\section{$N$-Persubstituierte 3,5-Diaminophenole und 1,3,5-Triaminobenzole und ihre Protonierung}

Von $F$. Effenberger und $R$. Niess ${ }^{[*]}$

$N$-Persubstituierte 3,5-Diaminophenole (2) und 1,3,5-Triaminobenzole (3) sind bisher noch nicht beschrieben worden. Verbindungen dieses Typs stellen als , aromatische Enamine ${ }^{\text {". }}$ interessante Substanzklassen dar. Durch Erhitzen von Phloroglucin (1) mit sek. Aminen im Autoklaven gelang uns die Darstellung der Aminobenzole (2) und (3) [11.<smiles>[R]N([R])c1cc(O)cc(N([R])[R])c1</smiles>
$\stackrel{\text { HNRR }^{\prime}}{\longrightarrow}$<smiles>[R]N([R])c1cc(N([R])[R])cc(N([R])[Z17])c1</smiles>

Die Umsetzungen erfolgen jeweils mit Aminüberschuß (Phloroglucin: Amin = 1:4), das Molverhältnis (2):(3) hängt nur von der Reaktionstemperatur ab. Durch fraktionierte Destillation oder Umkristallisieren lassen sich die Verbindungen reinigen.

\begin{tabular}{|c|c|c|c|c|c|}
\hline $\begin{array}{l}\text { Eingesetztes } \\
\text { Amin }\end{array}$ & $\begin{array}{l}\text { Temp. } \\
\left({ }^{\circ} \mathrm{C}\right)\end{array}$ & $\begin{array}{l}\text { Ausb. } \\
(\%)\end{array}$ & $\begin{array}{l}\text { (2) } \\
\mathrm{Fp}\left({ }^{\circ} \mathrm{C}\right) \\
(\mathrm{Kp}) \\
\left({ }^{\circ} \mathrm{C} / \text { Torr }\right)\end{array}$ & $\begin{array}{l}\text { Ausb. } \\
(\%)\end{array}$ & $\begin{array}{l}\text { (3) } \\
\text { Fp }\left({ }^{\circ} \mathrm{C}\right) \\
\text { (Kp) } \\
\left({ }^{\circ} \mathrm{C} / \text { Torr }\right)\end{array}$ \\
\hline Pyrrolidin & $\begin{array}{r}20 \\
180-200\end{array}$ & $\begin{array}{r}100 \\
-\end{array}$ & $174-185$ & $\overline{95}$ & $179-181$ \\
\hline Piperidin & $\begin{array}{l}180-200 \\
280-300\end{array}$ & $\begin{array}{l}98 \\
-\end{array}$ & $154-156$ & $\overline{40}$ & 184 \\
\hline Morpholin & $\begin{array}{l}180-200 \\
280-300\end{array}$ & $\begin{array}{l}91 \\
-\end{array}$ & $185-187$ & $\frac{-}{37}$ & $308-312$ \\
\hline $\begin{array}{l}\text { Dimethyl- } \\
\text { amin }\end{array}$ & $\begin{array}{r}130-150 \\
220\end{array}$ & $\begin{array}{l}68 \\
14\end{array}$ & $(148 / 0,008)$ & $\begin{array}{r}22 \\
69\end{array}$ & $(131 / 0,01)$ \\
\hline
\end{tabular}

Die Triaminobenzole (3) bilden mit Mineralsäure definierte Mono-, Di- oder Trisalze.

Bei der Monoprotonierung entstehen in Abhăngigkeit von den Substituenten $\mathbf{R}$ und $\mathbf{R}^{\prime}$ stabile $N$-oder $C$-Protonierungsprodukte (4) bzw. (5).

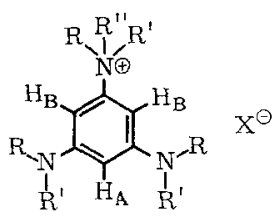

(4), $R^{\prime \prime}=\mathrm{H}$

(6), $\mathrm{R}^{\prime \prime}=\mathrm{CH}_{3}$

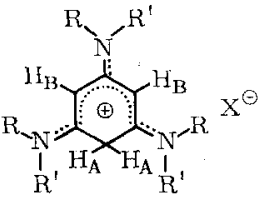

(5)
Tripiperidinobenzol $\left[(3), \mathbf{R}+\mathbf{R}^{\prime}=\left(\mathrm{CH}_{2}\right)_{5}\right]$ z.B. gibt $N$-Protonierung zu (4), während mit Tripyrrolidinobenzol [(3), $\left.\mathrm{R}+\mathbf{R}^{\prime}=\left(\mathrm{CH}_{2}\right)_{4}\right]$ kristalline Verbindungen des Typs (5) entstehen. (Physikalische Eigenschaften: z.B. (5), X $=$ BF $_{4}$ : gelbe Nadeln, die bei $254-257^{\circ} \mathrm{C}$ schmelzen.)

Die Strukturzuordnung gelingt NMR-spektroskopisch. So werden für das Tripyrrolidinobenzol-hydrojodid $[(5), \mathbf{R}+$ $\left.\mathrm{R}^{\prime}=\left(\mathrm{CH}_{2}\right)_{4}, \mathrm{X}=\mathrm{J}\right]$ in $\mathrm{DCCl}_{3} \mathrm{H}_{\mathrm{B}}$ als Singulett bei $\tau=5,17$ und $\mathrm{H}_{\mathrm{A}}$ ebenfalls als Singulett bei $\tau=6,20$ gefunden (relative Intensitäten 1:1).

Besondere Strukturverhältnisse sind auf Grund $\operatorname{der}^{1} \mathrm{H}-\mathrm{NMR}$ Spektren für Tripiperidinobenzol-monoperchlorat [(4),
$\left.\mathrm{R}+\mathrm{R}^{\prime}=\left(\mathrm{CH}_{2}\right)_{5}, \mathrm{X}=\mathrm{ClO}_{4}\right]$ anzunehmen. In polaren Lösungsmitteln, z. B. $\left(\mathrm{CD}_{3}\right)_{2} \mathrm{CO}$ oder $\left(\mathrm{CD}_{3}\right)_{2} \mathrm{SO}$, erscheinen die drei Kernprotonen als Singulett bei $\tau=3,6$, während das am Stickstoff gebundene Proton nur durch Integration zu erfassen ist, was für einen raschen Protonenübergang zwischen den Aminogruppen spricht. In $\mathrm{DCCl}_{3}$ dagegen ist dieses Proton an einer Aminofunktion fixiert, und die drei Kernprotonen sind im Spektrum extrem aufgespalten $(1 \mathrm{H}$ bei $\tau=2,10[\mathrm{~N}-\mathrm{H}], 2 \mathrm{H}$ bei $\tau=3,38$ und $1 \mathrm{H}$ bei $\tau=4,63$ ).

Eine meta-Kopplung der Ringprotonen $\mathrm{H}_{\mathrm{A}}$ und $\mathrm{H}_{\mathrm{B}}$ wird nicht beobachtet. Die vergleichbare $N$-Methylverbindung [(6), $\left.\mathbf{R}+\mathbf{R}^{\prime}=\left(\mathrm{CH}_{2}\right)_{5}, \mathrm{X}=\mathrm{ClO}_{4}\right]$ dagegen zeigt nur eine geringe Aufspaltung der Kernprotonen $(\tau$-Wert zentriert bei $\approx$ $3,5)$ und eine deutliche meta-Kopplung $\left(\mathrm{J}_{\mathrm{AB}}=2 \mathrm{~Hz}\right)$.

Aus den NMR-Spektren schließen wir auf eine starke elektronische Störung des aromatischen $\pi$-Elektronensystems im Tripiperidinobenzol-monoperchlorat. Eine Alternative zur benzoiden Form (4), die mit dem NMR-Spektrum in Einklang steht, ist eine valenzisomere „,Dewar-Struktur", für die jedoch sonst keine Beweise vorliegen.

Weitere Untersuchungen und insbesondere eine Röntgenstrukturanalyse des Tripiperidinobenzol-monoperchlorats sind in Arbeit.

Eingegangen am 11. August 1966, ergänzt am 9. September 1966 und 6. November 1967 [Z 320] Auf Wunsch der Autoren erst jetzt veröffentlicht

[*] Doz. Dr. F. Effenberger und Dipl.-Chem. R. Niess Institut für Organische Chemie der Universität 7 Stuttgart, Azenbergstraße 14/16

[1] Über die Darstellung der Aminobenzole haben wir anläßlich der Chemiedozenten-Tagung in Würzburg am 29. April 1966 berichtet. 